\title{
Relaciones de caso y extensión de la concordancia en el español peninsular y el portugués europeo
}

\author{
Case-marking and agreement in Peninsular Spanish \\ and European Portuguese
}

\author{
Víctor Lara Bermejo \\ Universität Bern \\ victor.lara@rom.unibe.ch
}

ORCID iD: https://orcid.org/0000-0002-0589-9495

\begin{abstract}
RESUMEN: El andaluz occidental y el portugués europeo cuentan con un solo pronombre plural en su sistema de tratamientos (ustedes/vocês), el cual puede inducir dos concordancias distintas: de segunda persona de plural (como su semántica) o de tercera persona de plural (como su sintaxis). Sin embargo, ninguno de los dos fenómenos ha sido investigado en profundidad, por lo que desconocíamos de qué dependía la adopción de una persona gramatical $u$ otra. En este artículo se pretende dar cuenta de la extensión de la concordancia en ambos casos y de los factores teóricos que llevan a dicho comportamiento.
\end{abstract}

Palabras clave: concordancia, andaluz, portugués europeo, persona, caso.

ABSTRACT: Western Andalusian and European Portuguese only rely on a single plural pronoun in their address system (ustedes/vocês) which can induce two different agreement types: (i) the second person plural (following the semantics of the address system) and (ii) the third person plural (following their syntax). These two agreement patterns have not been studied in depth and, consequently, we did not know what factors underlie this variation in agreement. In this paper, I study the linguistic spread of these agreement patterns as well as the theoretical factors that led to this variation in use.

Keywords: agreement, Andalusian, European Portuguese, person, case. 


\section{INTRODUCCIÓN}

El español peninsular estándar posee cuatro pronombres de tratamiento: dos para la informalidad (tú y vosotros, singular y plural respectivamente) y dos para la formalidad (usted y ustedes, singular y plural respectivamente). La formalidad se expresa mediante la tercera persona mientras que la informalidad opta por la segunda (Tabla 1).

\begin{tabular}{|l|c|c|}
\cline { 2 - 3 } \multicolumn{1}{c|}{} & Informalidad & Formalidad \\
\hline Singular & Tú $+2 \mathrm{sg}$ & Usted $+3 \mathrm{sg}$ \\
\hline Plural & Vosotros $+2 \mathrm{pl}$ & Ustedes $+3 \mathrm{pl}$ \\
\hline
\end{tabular}

TABLA 1.- Sistema de tratamiento en el español peninsular estándar

Sin embargo, la zona occidental de Andalucía eliminó esta distinción alrededor del siglo XVIII (Fernández Martín, 2012) y niveló en el plural cualquier tratamiento en el pronombre ustedes. No obstante, a pesar de que la sintaxis obliga a concordar ustedes en 3pl, dicho pronombre puede alternar con desinencias de 2pl y 3pl (Alvar, 1996; Cano, 2004 y 2008; Carrasco Santana, 2002; De Jonge y Nieuwenhuijsen, 2012; Lapesa, 1981; Menéndez Pidal, 2005; Penny, 2004 o Real Academia Española y Asociación de Acaemias de la Lengua Española, 2009). Esta situación es idéntica a la del español americano, con la única diferencia de que este último concuerda sistemáticamente ustedes en $3 \mathrm{pl}$ (Fontanella de Weinberg, 1999). La desaparición de vosotros en el español de América se ha fechado alrededor del siglo XIX, donde tan solo estaba relegado a la oratoria parlamentaria, del ejército o incluso eclesiástica (Vázquez Laslop, 2010), y aparecía casi de manera residual en obras literarias (Moreno de Alba, 2010).

En cuanto al andaluz occidental, los datos de que disponíamos hasta ahora (destacan los trabajos de Alvar, 1961-1965; Carricaburo, 1997; Lapesa, 2000 [1970] y Hummel, Kluge y Vázquez, 2010) tan solo sugerían que el reflexivo y el verbo en pretérito perfecto simple adoptaban la $3 \mathrm{pl}$, mientras que el resto de elementos lo hacían en $2 \mathrm{pl}$, excepto el posesivo que, en principio, se construía con el sintagma preposicional de ustedes (Tabla 2).

\begin{tabular}{|l|c|}
\cline { 2 - 2 } \multicolumn{1}{c|}{} & Concordancia \\
\hline Pronombre tónico & ustedes $(3 \mathrm{pl})$ \\
\hline Reflexivo & $3 \mathrm{pl}$ \\
\hline Pretérito perfecto simple & $3 \mathrm{pl}$ \\
\hline Otros tiempos verbales & $2 \mathrm{pl}$ \\
\hline Objetos & $2 \mathrm{pl}$ \\
\hline Posesivo & de ustedes \\
\hline
\end{tabular}

TABLA 2.-Concordancias de la nivelación en ustedes 
Lara (2012) mostró gracias a los datos del Atlas lingüístico de la Península Ibérica (ALPI) que la nivelación en ustedes discurría por las provincias de Huelva, Sevilla, Cádiz, Málaga (salvo el extremo oriental) y Córdoba (menos la parte septentrional) y que esta, efectivamente, podía inducir la $3 \mathrm{pl}$ o la $2 \mathrm{pl}$, según una jerarquía (i).

(i) Pronombre tónico $>$ reflexivo $>$ acusativo $>$ verbo subordinado

El contínuum representado en (i) muestra la extensión de la 3pl por los distintos elementos que hacen referencia a ustedes: si la $3 \mathrm{pl}$ aparece en el acusativo, forzosamente surge en el reflexivo y en el pronombre tónico, pero aún no en el verbo de la subordinada. En (1a-d) se muestra la evolución de la concordancia de acuerdo con los datos del $A L P I$.

(1) a. Ustedes no os disteis cuenta de cuándo os vieron mientras caminabais

b. Ustedes no se disteis cuenta de cuándo os vieron mientras caminabais

c. Ustedes no se disteis cuenta de cuándo los vieron mientras caminabais

d. Ustedes no se disteis cuenta de cuándo los vieron mientras caminaban

La metodología del ALPI, basada en un cuestionario con oraciones y palabras preestablecidas que los informantes tenían que repetir de acuerdo con su variedad, no proporcionó información sobre la concordancia que todos los elementos sintácticos adoptaban, ya que no hubo preguntas donde se incluyera un dativo, un posesivo u otros tiempos verbales. Asimismo, el atlas ofrece pocos datos cuantitativos, pues tan solo se recogía una respuesta por oración y de un solo informante (siempre varón, rural, sin estudios y con una edad mayor de 50) (Sanchís Guarner, 1962).

El portugués europeo presenta un caso análogo al del andaluz occidental. A pesar de que su sistema de tratamientos en plural se vio modificado en el siglo XVIII (Cintra, 1972; Faraco, 1996; Menon, 2006), actualmente el estándar opta por nivelar en vocês cualquier tratamiento de plural, si bien cuenta con muchos sintagmas nominales que expresan parentesco o diferenciación social y profesional, en caso de querer ser algo más cortés (Braun, 1988; Carreira, 2003). De entre todas esas fórmulas, la más usual y menos marcada se corresponde con os senhores/as senhoras y, al igual que vocês, ha de concordarse en 3 pl (Tablas 3 y 4).

\begin{tabular}{|c|c|}
\hline Informalidad & Formalidad \\
\hline Vós $+2 \mathrm{pl}$ & Vocês $+3 \mathrm{pl}$ \\
\hline
\end{tabular}

TABLA 3.- Sistema plural de tratamientos del portugués europeo antes del siglo XVIII 


\begin{tabular}{|c|c|c|}
\hline Informalidad & Formalidad & Distancia \\
\hline Vocês & Vocês & Os senhores / as senhoras \\
\hline
\end{tabular}

TABLA 4.- Sistema plural de tratamientos en el portugués europeo actual

No obstante, la propia norma obliga a optar por morfología de $3 \mathrm{pl}$ en ciertos elementos y morfología de $2 \mathrm{pl}$ en otros. Así, el reflexivo y cualquier tiempo verbal reciben $3 \mathrm{pl}$ mientras que los pronombres de objeto y el posesivo se concuerdan en 2pl (Tabla 5) (Cunha y Cintra, 1992; Mateus et al. 2006, Buzaglo et al. 2013).

\begin{tabular}{|l|c|}
\cline { 2 - 2 } \multicolumn{1}{c|}{} & Concordancia \\
\hline Pronombre tónico & Vocês $(3 \mathrm{pl})$ \\
\hline Reflexivo & $3 \mathrm{pl}$ \\
\hline Verbo & $3 \mathrm{pl}$ \\
\hline Objetos & $2 \mathrm{pl}$ \\
\hline Posesivo & $2 \mathrm{pl}$ \\
\hline
\end{tabular}

TABLA 5.-Concordancias de la nivelación en vocês

De nuevo, Lara (2012) investigó la realidad de este fenómeno en el ALPI y comprobó que el sistema estándar tan solo se daba en la mitad sur de Portugal, mientras que la mitad norte aún permanecía con el sistema antiguo, representado en la Tabla 3. Y al igual que con el andaluz occidental, la 3pl se extendía jerárquicamente de acuerdo con (ii).

(ii) Pronombre tónico / reflexivo / verbo $>$ acusativo

El contínuum expone que si la 3pl se manifiesta en el acusativo necesariamente aparece en el verbo, reflexivo y pronombre tónico (2a-b).

(2) a. Vocês não se aperceberam de quando vos Vosotros.2PL no REFL.3PL darse cuenta.3PL.PST. de cuando ACC.2PL. viram

ver.3PL.PST

('No os disteis cuenta de cuándo os vieron')

b. Vocês não se aperceberam de quando os

Vosotros.2PL no REFL.3PL darse cuenta.3PL.PST. de cuando ACC.3PL. viram

ver.3PL.PST

('No os disteis cuenta de cuándo os vieron')

Una vez más, los datos del ALPI no dilucidaron la situación del dativo o el posesivo y, como hemos explicado anteriormente, la metodología de este atlas no permite el análisis cuantitativo de un fenómeno, ya que el modus operandi 
pudo haber influido en la respuesta del encuestado. Por tanto, los datos que se recogen acerca de los resultados del ALPI han de evaluarse teniendo en cuenta las restricciones del propio método.

\section{CORPUS Y METODOLOGÍA}

Con el fin de dar cuenta de la realidad social y lingüística de ambos fenómenos en la actualidad y compensar las carencias de otros métodos, se ha llevado a cabo un trabajo de campo por Andalucía occidental y la parte centromeridional de Portugal. Dicho trabajo ha consistido en una serie de encuestas en las que los informantes tenían que visualizar varias escenas de dos comedias de situación conocidas, las cuales suelen mostrar a un personaje que se dirige a un grupo de personas. Tras la visualización, debían convertirse en el personaje y doblar la escena, de tal manera que pudieran producir tantas ocurrencias como fuera posible. Los informantes eran grabados mientras llevaban a cabo la actividad y, posteriormente, los archivos sonoros se transcribían (Lara, 2016). Cualquier ocurrencia que incluyera una segunda persona del plural se clasificaba de acuerdo con su función sintáctica (sujeto, reflexivo, objeto directo, objeto indirecto, posesivo, verbo) y con los rasgos extralingüísticos que caracterizaban al informante (género, edad, nivel educativo, lugar de origen, número de habitantes del lugar de origen). Asimismo, dentro de la categoría verbo, se discernía entre el tiempo y el modo verbales, y se añadía la información de la modalidad o el hecho de que se explicitara en una oración principal o subordinada y, en el caso de esta última, qué tipo de subordinada. Por último, la recopilación de escenas se basó en distintos tipos de interlocutores (amigos, familia, desconocidos,

\begin{tabular}{|l|c|c|}
\cline { 2 - 3 } \multicolumn{1}{c|}{} & Informantes & Ocurrencias \\
\hline Hombres & $129(48,3 \%)$ & $2156(44,6 \%)$ \\
\hline Mujeres & $139(51,7 \%)$ & $2676(55,4 \%)$ \\
\hline Total & $268(100 \%)$ & $4832(100 \%)$ \\
\hline
\end{tabular}

TABLA 6.-Número de informantes y ocurrencias del corpus (género)

\begin{tabular}{|l|c|c|}
\cline { 2 - 3 } \multicolumn{1}{c|}{} & Informantes & Ocurrencias \\
\hline$<30$ años & $104(38,8 \%)$ & $2103(43,5 \%)$ \\
\hline $30-60$ años & $104(38,8 \%)$ & $2072(42,9 \%)$ \\
\hline$>60$ años & $60(22,4 \%)$ & $657(13,6 \%)$ \\
\hline Total & $268(100 \%)$ & $4832(100 \%)$ \\
\hline
\end{tabular}

TABLA 7.--Número de informantes y ocurrencias del corpus (edad) 
personas mayores, etc.) con el fin de extraer posibles diferencias pragmáticas que se atestiguaran en la concordancia. A continuación, se expone el número de informantes y ocurrencias que este corpus dio como resultado (Tablas 6 a 9).

\begin{tabular}{|l|c|c|}
\cline { 2 - 3 } \multicolumn{1}{c|}{} & Informantes & Ocurrencias \\
\hline Con estudios superiores & $65(24 \%)$ & $1160(24,1 \%)$ \\
\hline Sin estudios superiores & $203(76 \%)$ & $3672(75,9 \%)$ \\
\hline Total & $268(100 \%)$ & $4832(100 \%)$ \\
\hline
\end{tabular}

TABLA 8.-Número de informantes y ocurrencias del corpus (nivel educativo)

\begin{tabular}{|l|c|c|}
\cline { 2 - 3 } \multicolumn{1}{c|}{} & Informantes & Ocurrencias \\
\hline$<5.000$ hab. & $38(11,5 \%)$ & $531(10,9 \%)$ \\
\hline $5.000-10.000$ hab. & $70(27,7 \%)$ & $1210(26,7 \%)$ \\
\hline $10.000-20.000$ hab. & $69(26 \%)$ & $1186(25,6 \%)$ \\
\hline $20.000-100.000$ hab. & $20(7,5 \%)$ & $201(5,6 \%)$ \\
\hline $100.000-500.000$ hab. & $45(17 \%)$ & $889(19,4 \%)$ \\
\hline$>500.000$ hab. & $26(10,3 \%)$ & $815(17,8 \%)$ \\
\hline Total 268 & $(100 \%)$ & $4832(100 \%)$ \\
\hline
\end{tabular}

TABLA 9.- Número de informantes y ocurrencias del corpus (población del enclave)

A los datos se les han aplicado dos fórmulas estadísticas: la prueba del chi cuadrado de Pearson así como una regresión logística. La primera da la significación real de una variable independiente (género, edad, etc.) y la última ordena el grado de afectación de cada variable significativa. Para los fenómenos que nos ocupan, el chi cuadrado ha resaltado la importancia de los factores edad, nivel educativo y tamaño de población del enclave. Así, tanto en zonas españolas como portuguesas, la tendencia a imitar el patrón estándar (la distinción entre vosotros y ustedes para el español, y la nivelación en vocês para el portugués) suele relacionarse con informantes de mediana edad y con un alto nivel educativo que, a su vez, viven en entornos urbanos (Lara, 2015). No obstante, el fin de este artículo es dar cuenta de la extensión lingüística de la concordancia en los elementos con referencia ustedes y vocês, por lo que pasaremos a describir y analizar los resultados al respecto. Los datos que exponemos a continuación son, por tanto, novedosos y reflejan nuevos avances en el conocimiento de ambos fenómenos.

\section{Resultados}

\subsection{El sujeto y la concordancia con el verbo}

Independientemente de los condicionantes sociales (que no analizaremos 
aquí), ambos fenómenos se caracterizan por tener un comportamiento idéntico en cuanto a su configuración sintáctica. Empecemos por el español.

El uso de ustedes ha producido tres zonas geográficas según la frecuencia de uso del fenómeno y su comportamiento gramatical (Mapa 1).

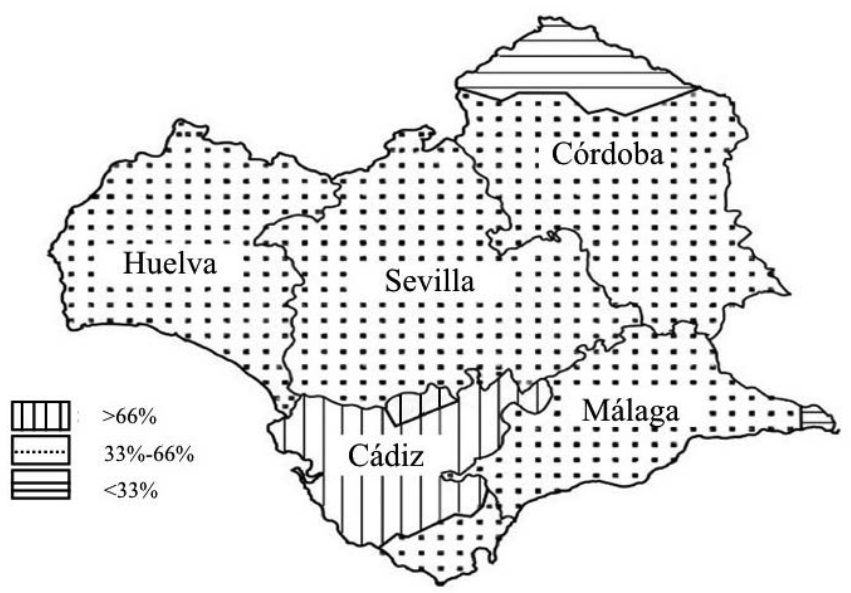

MAPA 1.-Extensión geográfica de la nivelación de ustedes en la actualidad

El Mapa 1 muestra que el área del centro-norte de Córdoba y el extremo este de Málaga se caracteriza por un uso bajo o nulo de ustedes como único tratamiento de plural. Dicho de otra forma, las zonas con un $<33 \%$, bien siguen el modelo estándar, bien cuentan con una baja proporción de hablantes que optan por la solución vernácula. En el lado opuesto hallamos el territorio comprendido por el sur de Sevilla y la provincia de Cádiz, con $>66 \%$ de empleo del fenómeno. En estas áreas, la práctica totalidad de hablantes optan por un solo pronombre y pocos se decantan por el patrón normativo del español peninsular. En una posición intermedia se encuentra la zona con 33\%-66\% de frecuencia de uso del fenómeno. Es decir, aquí encontramos la misma proporción de informantes que optan por el modelo estándar y la misma proporción de hablantes que se mantienen en el modelo dialectal.

Desde el punto de vista gramatical, las tres zonas presentan comportamientos distintos en lo que se refiere a la concordancia entre ustedes y el verbo. Mientras que el área $<33 \%$ se caracteriza por una fuerte discordancia (prácticamente todos los verbos adoptan la $2 \mathrm{pl}$ ), la zona $>66 \%$ ha regularizado la $3 \mathrm{pl}$ en el verbo de manera hegemónica. De nuevo, el terreno 33\%-66\% muestra un comportamiento intermedio, donde la $3 \mathrm{pl}$ es más numerosa que en la zona $<33 \%$ pero aún no es sistemática. 
La justificación de esta situación se debe a que ustedes no es el sujeto del verbo construido en $2 \mathrm{pl}$, sino el tópico, por lo que el verdadero sujeto es vosotros, el cual está elidido debido al carácter pro drop del español.

(3) Ustedes, no tenéis nómina

(4) Ustedes, habéis desorganizado mi casa

(5) Ustedes, no sois solventes

(6) Ustedes, estáis en paro

En los ejemplos (3-6), se observan distintas ocurrencias en las que los informantes pertenecientes a la zona $<33 \%$ han expresado una pausa entre el pronombre ustedes y el verbo. Como se ha comentado, esta ha sido la norma en dicha área (50 casos de 50 de ustedes expreso más verbo). Estas discordancias no son anómalas en el español ni en muchas otras lenguas, como han investigado Ackema y Neeleman (2013), Choi (2013) y Höhn (2016). De hecho, Ordóñez y Treviño (1999) o Fábregas (2008) (para el español) y Papangeli (2000) (para el griego) han estudiado el empleo de una concordancia verbal distinta a la del sujeto, como en (7) y (8).

(7) Los estudiantes somos jóvenes

(8) La gente somos muy curiosos

Si bien la amplia bibliografía al respecto se ha ocupado de sintagmas nominales (sobre todo en plural), apenas hay estudios sobre discordancias entre pronombres personales y tiempos verbales dependientes de estos. Uno de los fenómenos más llamativos del español a este respecto es el voseo, cuyas concordancias alternan entre las propias de vos y las de tú. Abadía de Quant (1992), Bertolotti y Coll (2003) y Fontanella de Weinberg (1979) argumenta que el uso de vos se inicia en el pronombre tónico y, posteriormente, va induciendo su concordancia de manera paulatina: primero, en el imperativo; segundo, en el presente de indicativo; y, por último, en el presente de subjuntivo (aunque de una forma muy desigual). Actualmente, el resto de tiempos verbales, así como los clíticos y el posesivo se construyen con morfología tuteante y no voseante.

Bosque y Gutiérrez Rexach (2009) justifican estas discordancias por cuestiones de topicalización, donde el elemento que se posiciona en la periferia izquierda no es realmente el sujeto que ha de concordar con el verbo, sino el tópico. Precisamente, las características de este son su comportamiento extraoracional, su autonomía y la obligatoriedad de ser recuperado anafóricamente dentro de la oración, sin que esa anáfora tenga que recibir obligatoriamente los mismos rasgos sintácticos que el tópico. Las construcciones de tópico han desencadenado cambios lingüísticos profundos tanto en español como en otras muchas lenguas. Elvira (1993 y 1996) y Fernández-Ordóñez (2009) explican que el orden actual 
del español SVO se debe a la frecuencia en etapa medieval con la que se colocaba en posición de tópico el sujeto de la oración; Adams (1987) explica que el francés medieval se comportaba como una lengua V2, pero la tendencia a ubicar en la periferia izquierda en posición de tópico el sujeto de la oración produjo el orden actual y la obligatoriedad de explicitarlo. El italiano presenta en la actualidad tres pronombres personales de tercera persona (lui, lei, loro) que, en su momento, eran oblicuos. La frecuencia con la que se colocaban en posición de tópico hizo que desplazaran los pronombres de sujeto normativos (egli, essa, essi) y se impusieran como nuevas formas de sujeto de tercera persona (Rohlfs, 1968 y Ernst et al. 2008).

Como ya se ha advertido, la particularidad del español es su carácter pro drop, por lo que la expresión del sujeto surge, sobre todo, en lecturas contrastivas o desambiguadoras. Por tanto, los ejemplos (3-6) carecen de un pronombre sintácticamente de $2 \mathrm{pl}$ (vosotros) porque estaría elidido. A este respecto, la Real Academia Española (RAE) (Real Academia Española y Asociación de Academias de la Lengua Española, 2009) afirma que la zona occidental de Andalucía presenta registros en los que se concatenan dos pronombres personales de $2 \mathrm{pl}$, como se reproduce en (9).

(9) Ustedes vosotros sois hermanos

El ejemplo (9) muestra efectivamente que la expresión de ambos pronombres en una misma oración existiría y que vosotros seguiría estando presente, al menos, en cierta zona del occidente andaluz. Sin embargo, la procedencia de (9) no se especifica ni se aclara nada más en cuanto a su idiosincrasia. A su vez, no aparece en ningún otro trabajo de corte dialectal o lingüístico, no se ha registrado en atlas del siglo pasado y, además, el corpus que presento en este artículo tampoco da cuenta de ocurrencias de estos dos pronombres tónicos expresos. El ejemplo que otorga la RAE, por tanto, tendrá que tratarse con cautela, ya que ignoramos en qué se fundamenta. No obstante, a pesar de que ninguna otra obra apoye la tesis sostenida por la RAE, mi corpus sí ha recogido la concatenación de los dos pronombres en otros contextos sintácticos (10-12).

(10) Se os queréis ir

(11) Intentarois entrar

(12) Me abrierois los grifos

Como se puede apreciar en (10-12), la explicitación de los dos pronombres en la misma oración es posible dialectalmente (se han registrado 32 ejemplos). En el primer caso, los informantes han expresado la forma reflexiva primero en $3 \mathrm{pl} \mathrm{y,} \mathrm{posteriormente,} \mathrm{en} 2 \mathrm{pl}$. Incluso la desinencia verbal muestra en su forma híbrida la aparición de ambas concordancias: -ro en 3pl e -is en $2 \mathrm{pl}$ (40 ejemplos de este caso). Es precisamente en el área 33\%-66\% donde estos registros se han recogido: la zona en la que el empleo de ustedes es intermedio y la 
concordancia sintáctica de $3 \mathrm{pl}$ es mayor que en la zona $<33 \%$, pero sigue sin ser sistemática, como en la $>66 \%$ (de 270 ejemplos de ustedes + verbo, 147 adoptan la $3 \mathrm{pl}$, y 123, la 2pl). Estos ejemplos indican que sería verosímil la existencia de dos pronombres tónicos expresos contiguos (ustedes + vosotros), la cual no se daría con asiduidad debido al carácter pro drop del español. La no necesidad de expresar el sujeto (vosotros) dejaría el tópico (ustedes) como único pronombre explícito, seguido de un verbo en $2 \mathrm{pl}$, el cual, en realidad, concordaría con vosotros y no con ustedes.

La incorporación de dos formas distintas que hacen referencia a la misma entidad dentro de la oración también se ha documentado históricamente en italiano. De nuevo, volviendo al desarrollo de los pronombres lui, lei y loro a costa de egli, essa, essi, se observa que en cierto período de este desarrollo, ambos pronombres convivieron dentro de la misma frase (si bien el uso antiguo había perdido peso fónico) (13-15).
(13) Lui, egli sa ogni cosa
3SG. 3SG. Saber-3SG.PRS cada cosa
(lit. 'Él, él sabe todo')
(14) Lui e' sa ogni cosa
3SG. 3SG saber-3SG.PRS cada cosa
(lit. 'Él él sabe todo')
(15) Lui sa ogni cosa
3SG. Saber-3SG.PRS cada cosa
(lit. 'Él sabe todo')

Como se puede ver, el uso de lui se inicia en construcciones de tópico, y es recuperado por una anáfora (egli). Con el tiempo, el tópico empieza a ser reinterpretado como sujeto, aunque aún no posee todos los rasgos propios de este y coexiste en la misma oración con el antiguo pronombre al que quiere sustituir. En última instancia, lui se acaba imponiendo y desplaza por completo el uso antiguo, que acaba desapareciendo. Esta última fase es exactamente la que hallamos en la zona $>66 \%$, donde el uso de ustedes es hegemónico, así como la concordancia en 3pl, como se observa en los ejemplos (16-20) (de 151 casos, 134 adoptaban la $3 \mathrm{pl}$ en el verbo y el resto, la $2 \mathrm{pl}$ ).

(16) Ustedes, ¿no estarían cotilleando?

(17) Son ustedes las que entraron en mi piso

(18) Ustedes me han pedido un crédito

(19) A ustedes, ¿qué les importa?

(20) Inundaron el piso

Las ocurrencias muestran una concordancia automática en 3pl debido al carácter de sujeto que ustedes posee en dicha zona, puesto que ha dejado de ser 
un tópico. Incluso en oraciones donde ustedes se explicita como tópico (16 y 19), la $3 \mathrm{pl}$ surge, ya que el sujeto es el mismo que el tópico. Es en esa área donde vosotros ya no existe y ustedes ha desplazado por completo el antiguo pronombre informal de 2pl. En la Tabla 10, se sintetiza el desarrollo de ustedes y la concordancia con el verbo.

\begin{tabular}{|l|c|c|c|}
\cline { 2 - 4 } \multicolumn{1}{c|}{} & Ustedes & Vosotros & Concordancia \\
\hline Fase $1(<33 \%)$ & Tópico & Sujeto & $2 \mathrm{pl}$ \\
\hline Fase $2(33 \%-66 \%)$ & Tópico-sujeto & Sujeto & $3 \mathrm{pl}+2 \mathrm{pl}$ \\
\hline Fase $3(>66 \%)$ & Sujeto & Eliminado & $3 \mathrm{pl}$ \\
\hline
\end{tabular}

TABLA 10.-Desarrollo de ustedes de tópico a sujeto

Esta evolución de tópico a sujeto está ampliamente documentada en la lingüística tipológica. Hopper y Traugott (2003) argumentan que los sujetos son básicamente tópicos reanalizados y que estos tienden a convertirse en sujetos porque se suelen colocar en posiciones que ocupan de manera prototípica (la periferia izquierda). Givón (1975 y 1990) es uno de los mejores exponentes del cambio de tópico a sujeto. De acuerdo con el autor, el desarrollo de un tópico a sujeto suele pasar tres fases distintas. En la primera (21), hallamos una construcción topical, donde el tópico se inserta en la periferia izquierda, seguida de una coma que marca la pausa prosódica con el resto de la oración, en la cual encontramos una anáfora que hace referencia a dicho tópico y que, en realidad, se comporta como el verdadero sujeto de la oración.

(21) The man, he came

El hombre 3SG. Venir.3SG.PST.

(lit. 'El hombre, él vino')

La frecuencia de la construcción (21) provoca que los hablantes reinterpreten el elemento ubicado a la izquierda como sujeto, ya que ocupa la posición prototípica de este. Sin embargo, antes de que sea completamente reanalizado como sujeto, el tópico entra en una fase intermedia en la que ya no posee todos los elementos de un tópico (desaparece la pausa y se inserta dentro de la oración), pero tampoco recibe todos los rasgos de un sujeto (necesita seguir siendo referenciado por una anáfora), como se muestra en el ejemplo (22).

(22) The man he came

El hombre 3SG. Venir.3SG.PST.

(lit. 'El hombre él vino') 
La última fase de este desarrollo se consuma cuando el hablante reinterpreta totalmente el antiguo tópico en el sujeto de la oración, provocando con ello la desaparición de la anáfora (23).
(23) The man
came
El hombre 3SG. Venir.3SG.PST.
(lit. 'El hombre vino')

Si aplicamos este proceso al fenómeno de la nivelación de ustedes, observamos que la fase ejemplificada en (21), se corresponde con la que se documenta en la zona $<33 \%$, donde ustedes actúa como tópico y es recuperado por una anáfora (vosotros) que es silent debido al carácter pro drop del español. Posteriormente, la fase ilustrada en (22) se documenta en el área 33\%-66\%, puesto que surgen ocurrencias donde las dos formas se expresan (se os, intentarois): una de ellas hace referencia al tópico y la otra, al verdadero sujeto. En este estadio, el tópico convive con el todavía sujeto, pero aún no goza del estatus de este, ya que necesita ser recuperado por una anáfora. Los casos en los que la forma que hace referencia al verdadero sujeto no aparece simplemente son silent. Por último, la fase reproducida en (23) se atestigua en el área $>66 \%$, donde ustedes es definitivamente un sujeto y, por tanto, induce concordancia de $3 \mathrm{pl}$ al verbo.

Las aparentes discordancias, como hemos expuesto, se han debido en realidad a elementos no expresos. La existencia de estos elementos que no se reproducen fonéticamente también ha sido ampliamente investigada. Ejemplo de ello para otras lenguas romances lo encontramos en Kayne (2003, 2005 y 2007). Según el autor, el francés y el italiano poseen construcciones que contravienen la norma o que muestran una aparente discordancia. Si prestamos atención al ejemplo (24) del italiano sobre construcciones reflexivas, observamos que el reflexivo no concuerda con el sujeto.
(24) Noi altri se
lavemo le man
1PL. REFL.3SG. lavar.1PL.PRS. las manos
(lit. 'Nosotros se lavamos las manos')

De acuerdo con Kayne, estas frases contienen un elemento silent que indica el rasgo de persona, pero que simplemente no se explicita fonéticamente. Así, (24) en realidad se corresponde con (25).
(25) Noi altri ne se lavemo le man
1PL. REFL.1PL. REFL.3SG. lavar.1PL.PRS. las manos
(lit. 'Nosotros nos se lavamos las manos')

Igualmente, el francés es propenso a construcciones de tópico, el cual se recupera seguidamente mediante una anáfora. Sin embargo, existen contraejemplos como los que se comparan en (26-29). 

(26) Lui (,)
a
téléphoné
3SG.MASC (3SG.MASC.CLIT) haber-3SG.PRES.IND telefonear-PCP ('Ha llamado por teléfono')
Eux (,) (ils)
ont
téléphoné
3PL.MASC (3PL.MASC.CLIT) haber-3PL.PRES.IND telefonear-PCP
('Han llamado por teléfono')
(28) Moi, *(j')
ai
téléphoné
1SG (1SG. CLIT) haber-1SG.PRES.IND telefonear-PCP
('He llamado por teléfono')
(29) Toi, *(tu) as téléphoné
2SG (2SG. CLIT) haber-2SG.PRES.IND telefonear-PCP
('Has llamado por teléfono')

De acuerdo con (26) y (27), los pronombres tónicos de tercera persona permiten la no explicitación del sujeto, frente al resto de personas, donde es obligatorio (28) y (29). La explicación para esto, siempre de acuerdo con Kayne, es la presencia de un elemento silent que sencillamente no se expresa.

En el caso del portugués, no hemos hallado discordancias entre vocês y verbo, por lo que inferimos que vocês es completamente un sujeto y no un tópico.

\subsection{Los clíticos y el posesivo}

Una vez que ustedes y vocês son completamente sujetos, empiezan a esparcir la concordancia sintáctica $(3 \mathrm{pl})$ en todos los elementos que hacen referencia a estas entidades. Su desarrollo es paulatino y progresivo, como observamos a continuación. Comencemos el análisis por los pronombres átonos o clíticos (Gráfico 1).

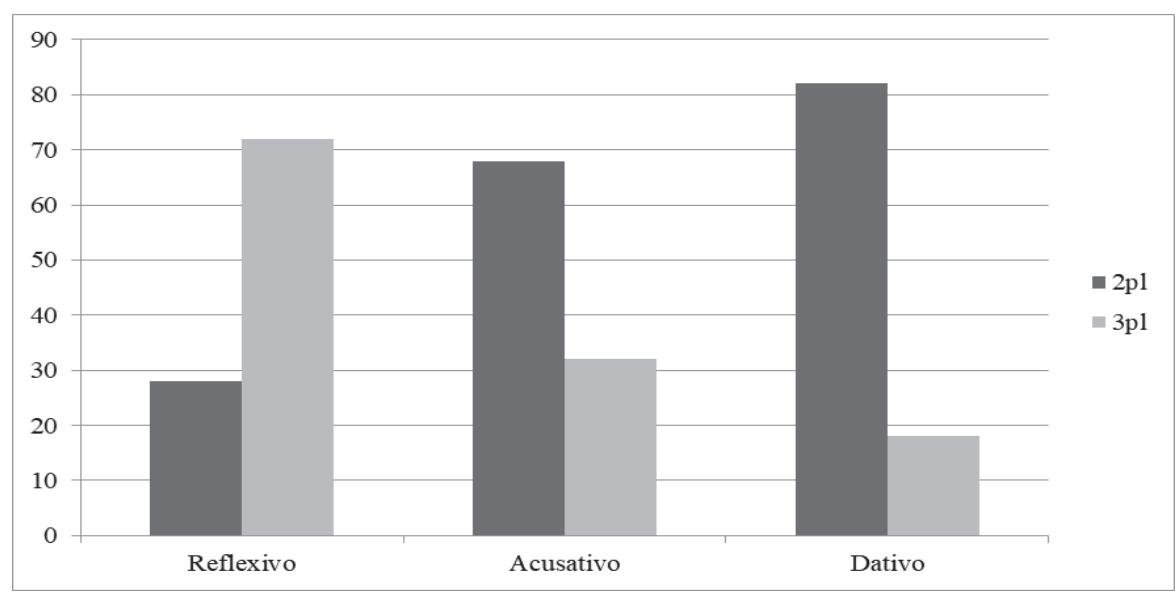

GRÁFICO 1.-Concordancia de los clíticos (ustedes) 
Como se observa en el Gráfico 1, el primer clítico que empieza a sucumbir a la $3 \mathrm{pl}$ es el reflexivo, seguido del acusativo y del dativo. De acuerdo con estos datos, hallamos tres fases representadas en (30-32).

(30) a. Ustedes se quedan en casa

b. (A ustedes) os vi pasear

c. (A ustedes) os doy un abrazo

(31) a. Ustedes se quedan en casa

b. (A ustedes) los vi pasear

c. (A ustedes) os doy un abrazo

(32) a. Ustedes se quedan en casa

b. (A ustedes) los vi pasear

c. (A ustedes) les doy un abrazo

En un primer estadio, el reflexivo adopta la 3pl (de 490 ejemplos, 352 adoptan la $3 \mathrm{pl}$ ), si bien los pronombres de objeto aún se construyen en 2 pl. En un estadio posterior, el acusativo toma la $3 \mathrm{pl}$ (de 190 casos, lo hacen 74) mientras el dativo todavía permanece reticente, ya que prefiere mantenerse con morfología de vosotros. En una fase ulterior, el dativo empieza a concordar en 3pl (de 349 casos, lo adoptan 59), por lo que todos los pronombres átonos terminan adoptando la concordancia que ustedes induce. Observemos el comportamiento del portugués a este respecto (Gráfico 2).

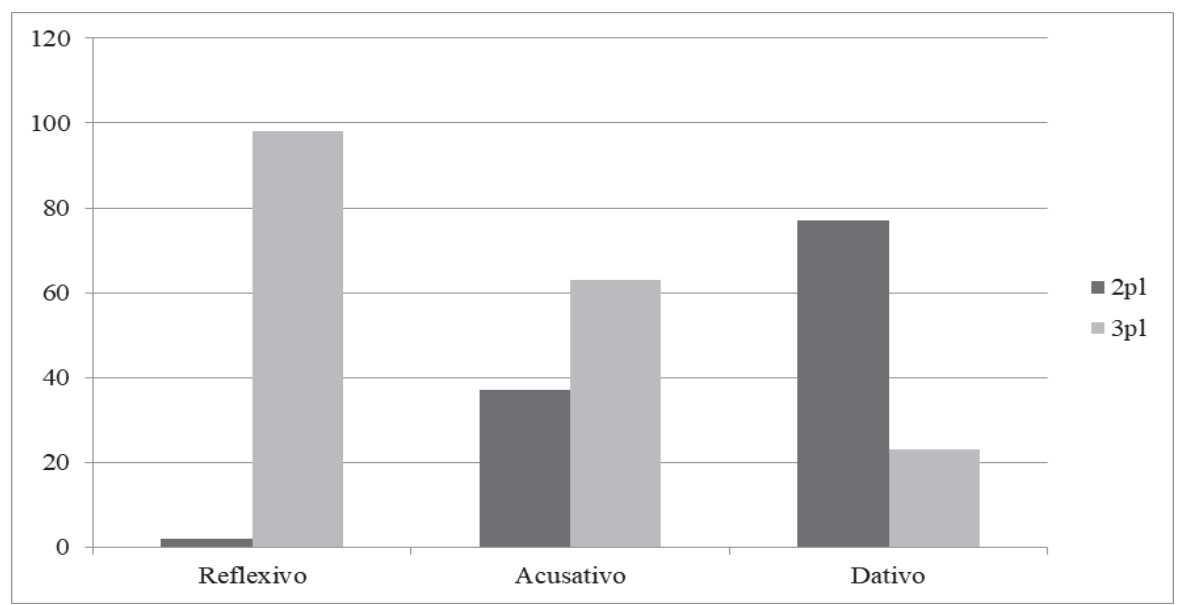

GRÁFICO 2.-Concordancia en los clíticos (vocês)

Si observamos el Gráfico 2, que engloba el porcentaje de uso de la 2pl o la $3 \mathrm{pl}$ en los clíticos, nos damos cuenta de que, efectivamente, el reflexivo se flexiona hegemónicamente en 3pl (de 65 ejemplos, lo han hecho 62), seguido 
por el acusativo (cuya alternativa en 2pl sube al 35\%: de 62 ejemplos, 42 han adoptado la $3 \mathrm{pl}$ ) y, el dativo, que presenta un porcentaje amplio de ocurrencias en $2 \mathrm{pl}$ (33-34) (de 52 ocurrencias, solo 14 se construyen en $3 \mathrm{pl}$ ). En el caso del reflexivo, las pocas ocurrencias de $2 \mathrm{pl}$ fueron con referencia al pronombre vós en áreas donde todavía permanece la antigua distinción diafásica entre vós y vocês, por lo que no hemos hallado discordancias entre vocês y el reflexivo.

(33) a. Ontem não os vi

ayer no ACC.3PL. ver.3PL.PST

('Ayer no os vi')

b. A mãe tem-vos contado alguma história?

La madre tener.3PL.PRS. DAT.2PL. contar.PCP. alguna historia

('Os ha contado mama una historia?)

(34) a. Ontem não os vi

ayer no ACC.3PL. ver.3PL.PST

('Ayer no os vi')

c. A mãe tem-lhes contado alguma história?

La madre tener.3PL.PRS. DAT.3PL. contar.PCP. alguna historia ('Os ha contado mama una historia?)

Terminemos con la situación del posesivo. Según el Gráfico 3, el sintagma preposicional de ustedes apenas se atestigua y es superado por la forma de $2 \mathrm{pl}$, vuestro, o la canónica de $3 \mathrm{pl}$, su, esta última en un $20 \%$.

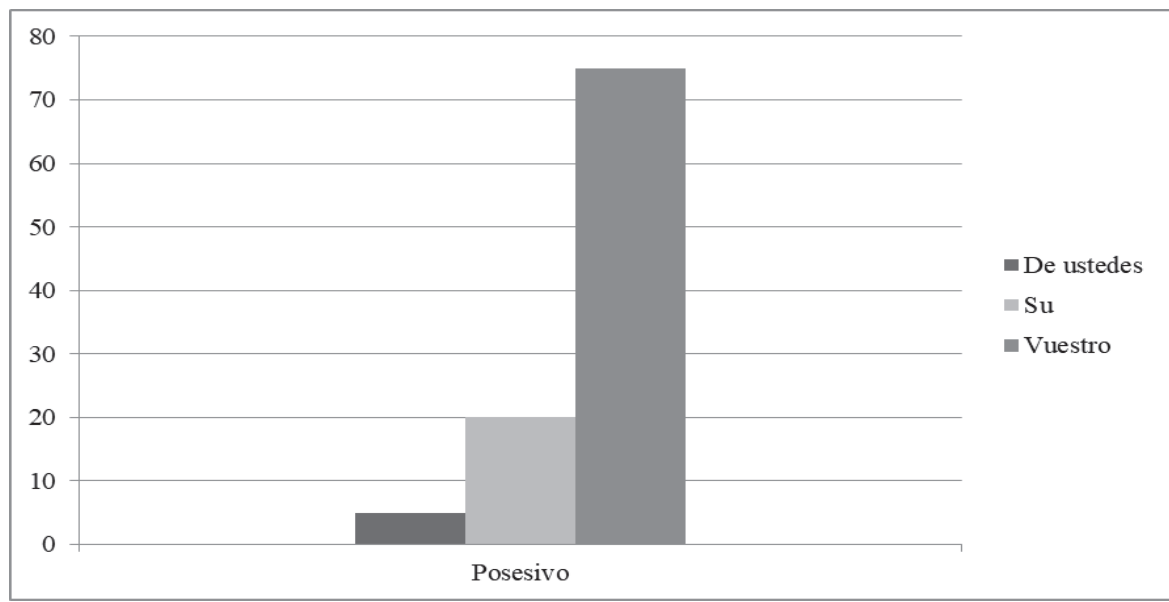

GRÁfICO 3.-Posesivo (ustedes)

Es precisamente en la zona donde la $3 \mathrm{pl}$ se ha extendido hasta el dativo (zona $>66 \%$ ) donde el posesivo empieza a concordar en 3pl, siendo así el último de la cadena en adaptarse a la nueva concordancia (de 155 ejemplos, tan 
solo 6 corresponden a de ustedes; 31 a su; y 118 a vuestro) (véanse los ejemplos de 35 a 41).

(35) Irse a sus casas

(36) Métanse en su vida

(37) No podemos hacernos cargo de su situación financiera

(38) Meterse en la vida de ustedes

(39) Meteros en vuestros asuntos

(40) Os vais a vuestra casa

(41) Estoy harto de vuestras tonterías

Este comportamiento de la concordancia puede sintetizarse en la jerarquía representada en (iii).

(iii) Sujeto $>$ reflexivo $>$ verbo $>$ acusativo $>$ dativo $>$ posesivo

Según este contínuum, si la $3 \mathrm{pl}$ surge en el dativo, forzosamente aparece en el acusativo, el verbo, el reflexivo y el sujeto. La extensión de la 3 pl recorre el contínuum de izquierda a derecha mediante fases implicativas.

En el caso del portugués, el posesivo empieza a construirse en 3 pl, aunque el espacio geográfico de dicha fase tan solo se documenta en el extremo suroriental de Portugal, en la frontera con el fenómeno del andaluz occidental de la nivelación en ustedes (de 50 ejemplos registrados, tan solo 9 han sido de $3 \mathrm{pl}$ ). Dichas fases se ejemplifican en (42-43) (Gráfico 4).

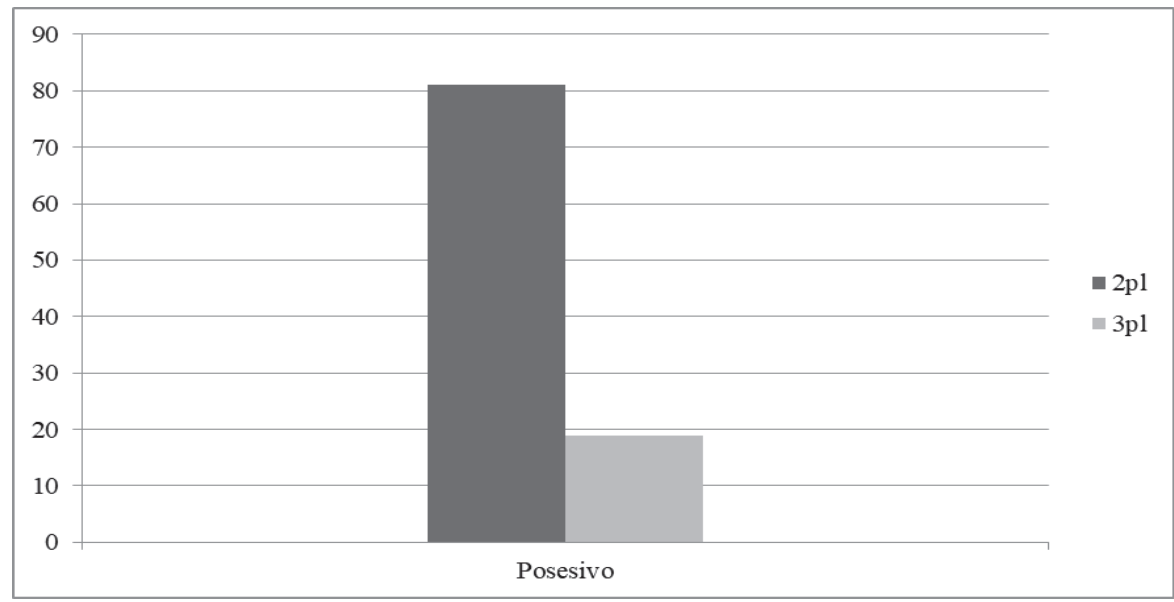

GRÁFICO 4.-Posesivos (vocês) 
(42) a. Como estão

os vossos pais?

Como estar.3PL.PRS. POSS.2PL padres

(‘¿Como están vuestros padres?’)

(43) a. Como estão os pais de vocês?

Como estar.3PL.PRS. los padres de vosotros.3PL

(‘¿Como están vuestros padres?’)

En resumen, el comportamiento lingüístico de la 3pl en el fenómeno nivelador de vocês puede resumirse en la jerarquía representada en (iv).

(iv) Sujeto / reflexivo / verbo $>$ acusativo $>$ dativo $>$ posesivo

El contínuum indica que si la $3 \mathrm{pl}$ surge en el acusativo, forzosamente aparecerá en los elementos de su izquierda, pero aún no en los de su derecha. La extensión por tanto de la $3 \mathrm{pl}$ recorre de izquierda a derecha la jerarquía, siempre en ese orden, y de una manera implicativa (Mapa 2).

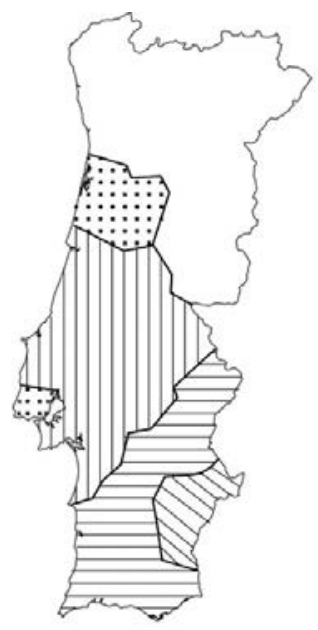

….... Fase 1: Sujeto+Reflexivo+Verbo

एD Fase 2: Sujeto+Reflexivo+Verbo+Acusativo

Fase 3: Sujeto+Reflexivo+Verbo+Acusativo+Dativo

$\Delta \nabla$ Fase 4: Sujeto+Reflexivo+Verbo+Acusativo+Dativo

+ Posesivo

MAPA 2.-Extensión geográfica de la nivelación en vocês en la actualidad

De acuerdo con el Mapa 2, actualmente el portugués europeo presenta cuatro fases. En la fase 1, el pronombre vocês ha desplazado a vós como tratamiento informal y ha generalizado la $3 \mathrm{pl}$ en el verbo y el reflexivo (44-45).

(44) Onde é que vocês se conheceram?

Donde es que vosotros.3PL REFL.3PL conocer.3PL.PST

(“¿Dónde os conocisteis?’) 

(45) Vocês estão a falar mal de alguém
Vosotros.3PL estar.3PL.PRS. a hablar mal de alguien
('Vosotros estáis hablando mal de alguien')

El tercer estadio supone la adopción de la $3 \mathrm{pl}$ en el acusativo, a pesar de la norma estándar de no incluir morfología de $3 \mathrm{pl}$ en los clíticos de objeto. Dicha fase se atestigua en zonas rurales del suroriente del Alentejo y en todo el Algarve. Inmediatamente después, el dativo adopta la 3pl, si bien su extensión es menor aún que la del acusativo.

Por último, el posesivo empieza a construirse en 3pl, aunque el espacio geográfico de dicha fase tan solo se documenta en el extremo suroriental de Portugal, en la frontera con el fenómeno del andaluz occidental de la nivelación en ustedes. La única zona que ha quedado aislada a las sucesivas oleadas innovadoras del fenómeno de vocês ha sido la ciudad de Lisboa, cuyo estatus de centro urbano, cultural, social y político ha provocado que se mantenga fiel a la regla estándar de no generalizar la 3 pl mas allá del reflexivo y el verbo.

En resumen, hemos visto que tanto el portugués como el español presentan un comportamiento idéntico en lo que respecta a la extensión de la concordancia de $3 \mathrm{pl}$ en los fenómenos de nivelación de ustedes y vocês. Empiezan como tópicos $\mathrm{y}$, una vez que son reinterpretados como sujetos, el reflexivo y el verbo empiezan a concordar. Posteriormente, son los pronombres de objeto (en concreto, con el orden acusativo-dativo) los que adoptan la innovación, la cual termina el recorrido en el posesivo, ya que, en ambas lenguas, resulta el último elemento en adoptar la $3 \mathrm{pl}$.

Cabe preguntarse por qué la extensión de la $3 \mathrm{pl}$ sigue ese patrón y no otro. De acuerdo con Corbett (2006), la concordancia depende de varios factores. En primer lugar, de la oposición controller y target. Mientras que el primero es el elemento inductor de la concordancia, el segundo es el elemento que la recibe. Así, si un controller induce dos concordancias distintas, el target adquirirá una de las dos de acuerdo con dos parámetros. Uno de ellos se refiere a la posición que tengan ambos dentro de la oración. Es decir, cuanto más alejados estén el controller y el target entre sí, mayor independencia tiene este para elegir la concordancia. Observemos el ejemplo (46), sacado de Corbett (2006).

(46) The committee has decided to pass the law El comité haber.3SG.PRS. decidir.PCP. PREP. aprobar.INF. la ley but they have been discussing the whole night pero ellos haber.3PL.PRS. estar.PCP. debatir.GER. la toda noche ('El comité ha decidido aprobar la ley, pero (ellos) han estado debatiendo toda la noche')

En (46), el controller (committee) induce concordancia singular como prueba el verbo, a pesar de que semánticamente sea plural. Sin embargo, su referencia se 
vuelve a recuperar en la adversativa mediante un pronombre en plural y un verbo que concuerda en plural con dicho pronombre. El empleo de they es reflejo de una mayor independencia, ya que el target se halla alejado del controller; de hecho, se encuentra en una oración distinta. Por eso, en esta ocasión su preferencia ha sido la concordancia semántica (plural) y no la opción sintáctica (singular).

El otro elemento del que depende la adopción de la concordancia se refiere al estatus que el target ostenta dentro de una jerarquía, representada en (v).

(v) Pronombre personal $>$ pronombre relativo $>$ predicado $>$ adjetivo adyacente

De acuerdo con el contínuum, cuanto más a la derecha nos desplacemos en la jerarquía, mayor probabilidad de que el target opte por una concordancia sintáctica, mientras que cuanto más a la izquierda, mayor es la posibilidad de que adopte una concordancia semántica. Observemos el ejemplo (47).

(47) Sus excelentísimas majestades están muy satisfechos con la noticia

En (47) advertimos que, aunque majestades es femenino, hace referencia a un masculino inclusivo. Los dos adjetivos que concuerdan con majestades adoptan tanto el masculino como el femenino, pero su opción depende del estadio del contínuum en (v). Mientras que excelentísimas se comporta como el adjetivo adyacente, satisfechos pertenece al ámbito del predicado y, por tanto, de acuerdo con la jerarquía, es más cercano a la concordancia semántica que el adjetivo adyacente. Prueba de este carácter alternante la tenemos en el neutro de materia. Fernández-Ordóñez (2006 y 2007) explica que la zona peninsular donde se da este fenómeno presenta una extensión de la concordancia que coincide con la jerarquía de Corbett, ya que la concordancia sintáctica surge en el adjetivo adyacente y se extiende, poco a poco, hasta el pronombre. Dicho contínuum va desde el centro-oriente de Asturias (donde la concordancia sintáctica está más arraigada) hasta la zona centro-occidental de Castilla, donde la concordancia semántica es más prolífica.

Por tanto, en el caso de ustedes y de vocês, el verbo y el reflexivo adoptan primero la concordancia sintáctica, ya que su cercanía y dependencia con respecto al controller es muy estrecha; solo cuando el pronombre inductor se comportan como un tópico la concordancia tiende a ser semántica, pues el controller ya no se halla en la misma oración. Los pronombres de objeto cuentan con mayor autonomía debido a que no poseen un controller que les induzca previamente una concordancia, sino que son la primera referencia a la entidad dentro de la frase (excepto en construcciones de topicalización o doblado). Por eso, tardan más en acceder a la 3pl, seguidos del posesivo, el cual también cuenta con suficiente autonomía (incluso más que los objetos) para adoptar una concordancia que no le es inducida por ningún controller. 
A pesar del enorme trabajo de Corbett, su investigación se limita a la diferencia sintáctica y semántica sobre todo en género y número, por lo que el conflicto de la persona gramatical $(2 \mathrm{pl}$ versus $3 \mathrm{pl})$ no queda resuelto en su estudio. La obra de Wechsler y Zlatic (2000 y 2003) aborda en mayor profundidad los conflictos de concordancia y distinguen para ello dos términos: index y concord. La concordancia index es aquella que se establece entre sujeto y predicado y está supeditada a los rasgos de persona, número, género (y a veces, caso); concord configura la concordancia dentro de un sintagma nominal y suele atender a los rasgos de caso, género y número.

Sin embargo, como Wechsler y Hahm (2011) argumentan, los pronombres de tratamiento a veces se caracterizan por un comportamiento totalmente independiente, como se desprende de los ejemplos (48-51).
(48) Vous êtes
loyal
Usted ser.2PL.PRS. fiel.SG.MASC.
('Usted es fiel')
(49) Vous êtes loyale
Usted ser.2PL.PRS. fiel.SG.FEM.
('Usted es fiel')
(50) Vous êtes loyaux
Usted ser.2PL.PRS. fiel.PL.MASC.
('Ustedes / vosotros son / sois fieles')
(51) Vous êtes loyales
Usted ser.2pL.PRS. fiel.PL.FEM.
('Ustedes / vosotras son / sois fieles')

Aunque en todos los casos vous induzca plural, parte de la concordancia index opta por el singular como única manera de desambiguar el referente. A pesar de que ustedes y vocês sean formas de tratamiento, la concordancia que inducen sí parece cumplir los principios de Wechsler y Zlatic (2000 y 2003). Por tanto, de acuerdo con estos autores, el reflexivo y el verbo deben aceptar los rasgos de concordancia que el sujeto les transmite, por lo que tanto ustedes como vocês, cuando son reanalizados como sujetos, tienen que inducir la $3 \mathrm{pl}$ en ambos elementos, ya que se establece una concordancia index que obliga a concertar el sujeto con el predicado. Los clíticos de objeto se comportan, en cambio, de manera más independiente. No suelen estar gobernados por una entidad que se exprese previamente, como en los casos de las dislocaciones de objeto. Casi siempre presentan por primera vez la referencia en la oración. Ya que los objetos no se refieren necesariamente a ninguna otra entidad expresada en la oración, no están obligados a concordar con nada más dentro del dominio oracional, es decir, no están obligados a adoptar concordancia index. El posesivo no obstante suele construirse dentro del sintagma nominal, por lo que es el elemento con menor presión para adoptar la marca de persona (ya que su concordancia es concord y no index). 
Independientemente del tipo de concordancia que adopten los elementos sintácticos dependientes de ustedes y vocês (sintáctica-semántica, index-concord), ambas se rigen por el mismo patrón en cuanto a los casos gramaticales (Tabla 11).

\begin{tabular}{|l|c|c|c|c|c|c|}
\cline { 2 - 7 } \multicolumn{1}{c|}{} & Sujeto & Reflexivo & Verbo & Acusativo & Dativo & Posesivo \\
\hline Fase 1 & $3 \mathrm{pl}$ & $2 \mathrm{pl} / 3 \mathrm{pl}$ & $2 \mathrm{pl} / 3 \mathrm{pl}$ & $2 \mathrm{pl}$ & $2 \mathrm{pl}$ & $2 \mathrm{pl}$ \\
\hline Fase 2 & $3 \mathrm{pl}$ & $3 \mathrm{pl}$ & $2 \mathrm{pl} / 3 \mathrm{pl}$ & $2 \mathrm{pl}$ & $2 \mathrm{pl}$ & $2 \mathrm{pl}$ \\
\hline Fase 3 & $3 \mathrm{pl}$ & $3 \mathrm{pl}$ & $3 \mathrm{pl}$ & $2 \mathrm{pl}$ & $2 \mathrm{pl}$ & $2 \mathrm{pl}$ \\
\hline Fase 4 & $3 \mathrm{pl}$ & $3 \mathrm{pl}$ & $3 \mathrm{pl}$ & $3 \mathrm{pl}$ & $2 \mathrm{pl}$ & $2 \mathrm{pl}$ \\
\hline Fase 5 & $3 \mathrm{pl}$ & $3 \mathrm{pl}$ & $3 \mathrm{pl}$ & $3 \mathrm{pl}$ & $3 \mathrm{pl}$ & $2 \mathrm{pl}$ \\
\hline Fase 6 & $3 \mathrm{pl}$ & $3 \mathrm{pl}$ & $3 \mathrm{pl}$ & $3 \mathrm{pl}$ & $3 \mathrm{pl}$ & $3 \mathrm{pl}$ \\
\hline
\end{tabular}

TABLA 11.-Extensión de la concordancia en ustedes y vocês

La Tabla 11 muestra que el primer elemento en concordar es el sujeto (y el término de sintagma preposicional de manera análoga al contar con la misma forma para ambas funciones) y aquellos estrechamente ligados a este: reflexivo y verbo (en el caso del español, observamos que primero concuerda el reflexivo y, posteriormente, el verbo, mientras que el portugués no ha ofrecido casos de discordancia entre sujeto, verbo y reflexivo). Es decir, aquellas funciones propias del nominativo o que refieren al mismo son las primeras en ceder ante la $3 \mathrm{pl}$. A estas le siguen el acusativo, el dativo y el genitivo, cuya función suele estar representada por el posesivo. Este orden no parece aleatorio, como se observa en (vi).

(vi) Nominativo $>$ acusativo $>$ dativo $>$ ablativo $>$ genitivo

Según Pinkster (1985 y 1990), el orden usual en latín con respecto a sus casos seguía una jerarquía que se correspondía con la representada en (vi). Es exactamente la misma por la que se rige la extensión de la concordancia (cabe recordar otra vez que, en el caso del español y el portugués, el término del sintagma preposicional es homófono con el sujeto y, por tanto, se acopla inmediatamente a los cambios de este, por lo que la fase del ablativo no es pertinente en este fenómeno). Además, dicha jerarquía de casos coincide con la jerarquía de funciones sintácticas a la que muchas lenguas obedecen en su propia configuración. Observemos (vii).

(vii) Sujeto $>$ objeto directo $>$ objeto indirecto $>$ oblicuos

El contínuum representado en (vii) muestra, de acuerdo con Blake (1994), que la mayoría de las lenguas sigue un orden no marcado basado en esta jerar- 
quía. Incluso la capacidad de producir una oración pasiva sigue dicho patrón. Mientras que el español tan solo tiene la posibilidad de pasivizar el objeto directo (52a-b), el inglés lo hace también con el objeto indirecto, lo cual supone forzosamente que produce una pasiva de un objeto directo (53a-b).

(52) a. El dinero te fue dado

b. *Tú fuiste dado el dinero
a. The money was given to you
el dinero ser.3SG.PST dar.PCP. a 2SG.
(lit. 'El dinero fue dado a ti')
b. You were given the money
2SG ser.2SG.PST. dar.PCP. el dinero
(lit. 'Tú fuiste dado el dinero')

Esta distinción en función del caso también la podemos ver en otros fenómenos universales. Keenan y Comrie (1977) apuntan que la capacidad de relativizar un elemento depende de la función de caso que tenga. Así, hay lenguas capaces de relativizar tan solo el sujeto, mientras otras lo hacen con el sujeto y el complemento directo, y otras con estas dos funciones más el objeto indirecto. No obstante, no hay lengua capaz de relativizar el objeto indirecto y no el directo y el sujeto. Por tanto, según (vii), toda lengua capaz de relativizar un objeto oblicuo puede hacerlo con los de su izquierda. Pero incluso el cambio de las valencias responde a este criterio. Comrie (1976 y 1989) arguye que la causativización en turco sigue esta jerarquía en la adición de valencias. Por ejemplo, si a una oración intransitiva se le añade otra valencia, el antiguo sujeto pasa a ser objeto directo y la nueva valencia, en sujeto; si se le añade otra más, el objeto directo se convierte en objeto indirecto, el antiguo sujeto en objeto directo y la nueva valencia en sujeto y, así, sucesivamente (54-55).

(54) a. Hasan öl-dü

Hasan.NOM morir-PST.

('Hasan ha muerto')

b. Ali Hasan-1 öl-dür-dü

Ali.NOM Hasan.ACC morir.CAUS.PST.

('Ali ha matado a Hasan')

(55) a. Müdür mektub-u imzala-dı

director.NOM carta.ACC firmar.PST

('El director firmó la carta')

b. Ali mektub-u müdür-e imzala-t-tı

Ali.NOM carta.ACC director.DAT firmar.CAUS.PST

('Ali ha hecho que el director firme la carta')

REVISTA DE FILOLOGÍA ESPAÑOLA (RFE), XCVIII, 1. ${ }^{\circ}$, enero-junio, 2018, pp. 85-110

ISSN 0210-9174, eISSN 1988-8538, https://doi.org/10.3989/rfe.2018.04 
Por tanto, si aplicamos las investigaciones de Blake (1994) o Keenan y Comrie (1977) a la extensión de la 3pl, observamos que efectivamente se da primero en función de sujeto y en los elementos que dependen de este o hacen referencia a él (reflexivo y verbo); posteriormente pasa al objeto directo (función prototípica del acusativo), después al indirecto (el caso común del dativo) y por último al posesivo (usualmente el genitivo). Si bien Blake explica que los casos oblicuos son previos en la jerarquía con respecto al genitivo, el caso de ustedes y vocês se caracteriza por tener la misma forma como pronombre tónico, sin distinción en la función de sujeto o término de sintagma preposicional, por lo que, una vez se constituye en el sujeto, el oblicuo automáticamente cede ante la 3 pl por tener exactamente la misma forma gramatical: ustedes y vocês.

\section{CONCLUSIONES}

El trabajo de campo ha dado como resultado la posibilidad de analizar detalladamente el comportamiento lingüístico de la nivelación de ustedes, en español, y de vocês, en portugués. Según los datos, los dos fenómenos se caracterizan por un comportamiento análogo. Una vez que ustedes y vocês son reanalizados como sujetos, la $3 \mathrm{pl}$ se extiende al resto de elementos sintácticos de manera jerárquica, siendo el reflexivo y el verbo los primeros en adoptarla (en el caso del español), seguidos en este orden por el objeto directo, el objeto indirecto y el posesivo. Para Corbett (2006), la adopción de una concordancia depende, entre otras cosas, de la independencia que el target tenga con respecto a su controller. En el caso que nos ocupa, el verbo y el reflexivo dependen directamente del sujeto, por lo que su independencia con el controller es mínima y, por ello, se acoplan antes a la 3pl. Los objetos y el posesivo, en cambio, no dependen de un elemento inductor, sino que son, por regla general, la primera referencia que se da de una entidad, lo cual les otorga autonomía suficiente para no concordar con ustedes o vocês automáticamente. De acuerdo con las investigaciones de Wechsler y Zlatic (2003), el verbo y reflexivo adoptan antes la $3 \mathrm{pl}$ porque son concordancia index y han de recoger los rasgos de persona, género y número del sujeto; los objetos no dependen del sujeto u otro inductor, por lo que no están tan obligados a construirse bajo los mismos parámetros que el verbo y el reflexivo, mientras que el posesivo atiende a los rasgos de caso, género y número, ya que suele insertarse en sintagmas nominales $\mathrm{y}$, por tanto, se caracteriza por la concordancia concord. Por último, observamos que la extensión coincide con los estudios realizados por Blake (1994) con respecto a las relaciones de caso y funciones sintácticas, ya que muchos fenómenos lingüísticos obedecen a la jerarquía ejemplificada en el artículo, desde la relativización, pasando por la pasivización o el orden de palabras no marcado. 


\section{BIBLIOGRAFÍA}

Abadía de Quant, Inés (1992): "La relación pronominal-verbal de segunda persona singular en el español de Corrientes durante el siglo XIX, su comparación con la situación en Buenos Aires", Revista Argentina de Linguística, 8, pp. 31-46.

Ackema, Peter y Ad Neeleman (2013): "Subset controllers in agreement relations", Morphology, 23, pp. 291-323.

Adams, Marianne (1987): "From old French to the theory of pro-drop", Natural Language and Linguistic Theory, 5, 1, pp. 1-32.

Alvar, Manuel (1961-1965): Atlas lingüístico y etnográfico de Andalucía (ALEA), Antonio Llorente y Gregorio Salvador (colabs.), Granada, Universidad de Granada.

Alvar, Manuel (1996): Manual de dialectología hispánica, Barcelona, Ariel.

Bertolotti, Virginia y Magdalena Coll (2003): "A synchronical and historical view of the tú/vos option in the Spanish of Montevideo", en Silvina Montrul y Francisco Ordóñez (eds.), Linguistic theory and language development in Hispanic languages, Somerville, Cascadilla Press, pp. 1-12.

Blake, Barry J. (1994): Case, Cambridge, Cambridge University Press.

Bosque, Ignacio y Javier Gutiérrez-Rexach (2009): Fundamentos de sintaxis formal, Madrid, Akal.

Braun, Friederike (1988): Terms of address. Problems of patterns and usage in various languages and cultures, Berlin, Mouton de Gruyter.

Buzaglo Paiva Raposo, Eduardo, Maria Fernanda Bacelar do Nascimento, Maria Antónia Coelho da Mota et al. (coord.) (2013): Gramática do português, Lisboa, Fundação Calouste Gulbenkian.

Cano, Rafael (2004): Historia de la lengua española, Barcelona, Ariel.

Cano, Rafael (2008): El español a través de los tiempos, Madrid, Arco/Libros.

Carrasco Santana, Antonio (2002): Los tratamientos en español, Salamanca, Ediciones Colegio de España.

Carreira, Maria Helena Araújo (2003): "Les formes allocutives en portugais européen: évolution, valeurs et fonctionements discursifs", Franco-British Studies, 33-34, pp. 35-45.

Carricaburo, Norma (1997): Las fórmulas de tratamiento en el español actual, Madrid, Arco/Libros.

Choi, Jaehoon (2013): "Pro-drop in pronoun-noun constructions", en Stefan Keine y Shayne Sloggett (eds.), NELS 42: Proceedings of the 42nd meeting of the North East Linguistic Society, Amherst, GLSA, pp. 119-128.

Cintra, Luís Felipe Lindley (1972): Sobre "formas de tratamento" na língua portuguesa, Lisboa, Horizonte.

Comrie, Bernard (1976): "The syntax of causative constructions: cross-language similarities and divergences", en Masayoshi Shibatani (ed.), The grammar of causative constructions (Syntax and Semantics 6), New York, Academic Press, pp. 261-312.

Comrie, Bernard (1989): Language universals and linguistic typology, Oxford, Blackwell.

Corbett, Greville (2006): Agreement, Cambridge, Cambridge University Press.

Cunha, Celso y Luís Felipe Lindley Cintra (1992): Nova gramática do português contemporâneo, Lisboa, João Sá de Costa.

De Jonge, Bob y Dorien Nieuwenhuijsen (2012): "Forms of address", en José Ignacio Hualde, Antxon Olarrea y Erin O'Rourke (eds.), The handbook of Hispanic linguistics, Malden, Blackwell, pp. 247-262.

Elvira, Javier (1993): "La función cohesiva de la posición inicial de frase en la prosa alfonsí", Cahiers de linguistique hispanique médiévale, 18/19, pp. 243-278.

Elvira, Javier (1996): "La organización del párrafo alfonsí", Cahiers de linguistique hispanique médiévale, 21, pp. 325-342. 
Ernst, Gerhard, Martin-Dietrich Gleßgen, Christian Schmitt y Wolfgang Schweickard (eds.) (2008): Romanische Sprachgeschichte, Berlin/New York, Mouton de Gruyter.

Fábregas, Antonio (2008): "Variación en forma morfológica de los pronombres de primera y segunda persona del plural", Revista Española de Linguística, 38, pp. 155-184.

Faraco, Carlos Alberto (1996): "O tratamento você em português: uma abordagem histórica", Fragmenta, 13, pp. 51-82.

Fernández Martín, Elisabeth (2012): La oposición vosotros/ustedes en la historia del español peninsular (1700-1931), Granada, Universidad de Granada.

Fernández-Ordóñez, Inés (2006): "Del Cantábrico a Toledo: el neutro de materia hispánico en un contexto románico y tipológico (I)", Revista de Historia de la Lengua Española, 1, pp. 67-118.

Fernández-Ordóñez, Inés (2007): "Del Cantábrico a Toledo: el neutro de materia hispánico en un contexto románico y tipológico (II)", Revista de Historia de la Lengua Española, 2, pp. 29-81.

Fernández-Ordóñez, Inés (2009): "Orden de palabras, tópicos y focos en la prosa alfonsî", Alcanate, VI, pp. 139-172.

Fontanella de Weinberg, Beatriz (1979): "La oposición cantes/cantés en el español de Buenos Aires", Thesaurus, XXXIV, 1, 2 y 3, pp. 72-83.

Fontanella de Weinberg, Beatriz (1999): "Sistemas pronominales de tratamiento usados en el mundo hispánico", en Ignacio Bosque y Violeta Demonte (dirs.), Gramática descriptiva de la lengua española, vol. 1, Madrid, Espasa, pp.1399-1425.

Givón, Talmy (1975): “Topic, pronoun and grammatical agreement”, en Charles Li (ed.), Subject and topic, New York, Academic Press Inc., pp. 149-188.

Givón, Talmy (1990): Syntax, a functional-typological introduction, Amsterdam/Philadelphia, John Benjamins Publishing Company.

Höhn, Georg F. K. (2016): "Unagreement is an illusion: Apparent person mismatches and nominal structure", Natural Language and Linguistic Theory, 34, 2, pp. 543-592. doi: <https:// doi.org/10.1007/s11049-015-9311-y>.

Hopper, Paul y Elizabeth Traugott (2003): Grammaticalization, Cambridge, Cambridge University Press.

Hummel, Martin, Bettina Kluge y M. ${ }^{a}$ Eugenia Vázquez Laslop (2010): Formas y fórmulas de tratamiento en el mundo hispánico, México DF, El Colegio de México/Karl-Franzens-Universität Graz.

Kayne, Richard (2003): "Person morphemes and reflexives in Italian, French and related languages", en Christina Tortora (ed.), The syntax of Italian dialects, Oxford, Oxford University Press, pp. 102-136.

Kayne, Richard (2005): Movement and silence, Oxford, Oxford University Press.

Kayne, Richard (2007): Some silent first person plurals, New York, New York University.

Keenan, Edward L. y Comrie, Bernard (1977): "Noun phrase accessibility and universal grammar", Linguistic Inquiry, 8, pp. 63-99.

Lapesa, Rafael (1981): Historia de la lengua española, Madrid, Gredos.

Lapesa, Rafael (2000) [1970]: Estudios de morfosintaxis histórica del español, Madrid, Gredos.

Lara, Víctor (2012): "Ustedes instead of vosotros and vocês instead of vós: an analysis through the Linguistic Atlas of the Iberian Peninsula (ALPI)", Dialectologia, Special Issue 3, pp. 57-93.

Lara, Víctor (2015): "Allocutive pronouns in Andalusia and their tendency toward standardisation”, Dialectologia, Special Issue 5, pp. 241-260.

Lara, Víctor (2016): "Spontaneous dubbing as a tool for eliciting linguistic data: the case of second person plural inflections in Andalusian Spanish", en Marie-Hélène Côté, Remco Knooihuizen y John Nerbonne (eds.), The future of dialects: selected papers from Methods $X V$, Berlin, Language Science Press, pp. 261-281.

Mateus, Maria Helena Mira, Ana Maria Brito, Inês Duarte et al. (2006): Gramática da língua portuguesa, Lisboa, Caminho. 
Menéndez Pidal, Ramón (2005): Historia de la lengua española, Madrid, RAE/Fundación Menéndez Pidal.

Menon, Odete Pereira da Silva (2006): "A história de você", en Marymarcia Guedes, Rosane de Andrade Berlinck y Clotilde de Almeida Azevedo Murakawa (orgs.), Teoria e análise lingüísticas: novas trilhas, Araraquara (São Paulo), Cult. Acadêmica, pp. 99-160.

Moreno de Alba, José (2010): "Notas sobre la cronología de la eliminación de vosotros en Amé-

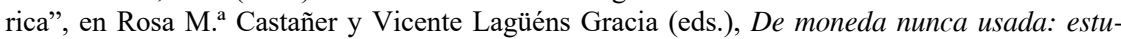
dios dedicados a José María Enguita Utrilla, Zaragoza, CSIC, pp. 461-470.

Ordóñez, Francisco y Esthela Treviño (1999): "Left dislocated subjects and the pro drop parameter: a case study of Spanish", Lingua, 107, pp. 39-68.

Papangeli, Dimitra (2000): "Clitic doubling in Modern Greek: a head-complement relation", en Corinne Iten y Ad Neeleman (eds.), Ucl working papers in linguistics, London, University College London, vol. 12, pp. 473-497.

Penny, Ralph (2004): Variación y cambio en español, Madrid, Gredos.

Pinkster, Harm (1985): "Latin cases and valency grammar: some problems", en Christian Touratier (ed.), Syntaxe et Latin: actes du IIème Congrès International de Linguistique Latine, Aixen-Provence, Université de Provence, pp. 163-190.

Pinkster, Harm (1990): Latin syntax and semantics, London, Routledge.

Real Academia Española y Asociación de Academias de la Lengua Española (2009): Nueva gramática de la lengua española, Madrid, Espasa.

Rohlfs, Gerhard (1968): Grammatica storica della lingua italiana e dei suoi dialetti, Torino, Einaudi.

Sanchís Guarner, Manuel (1962): "El Atlas Lingüístico de la Península Ibérica (ALPI). Trabajos, problemas y métodos", en Actas del IX Congreso Internacional de Lingüística Románica, Lisboa, Universidade de Lisboa, pp. 113-120.

Vázquez Laslop, M. ${ }^{a}$ Eugenia (2010): "Formas de tratamiento en el español de México", en Martin Hummel, Bettina Kluge y M. ${ }^{a}$ Eugenia Vázquez Laslop (eds.), Formas y fórmulas de tratamiento en el mundo hispánico, México DF, El Colegio de México/Karl-Franzens-Universität Graz, pp. 247-269.

Wechsler, Stepehen y Hyun-Jong Hahm (2011): "Polite plurals and adjective agreement", Morphology, 21, pp. 247- 281.

Wechsler, Stephen y Larissa Zlatic (2000): "A theory of agreement and its application to SerboCroatian”, Language, 76, pp. 799-832.

Wechsler, Stephen y Larissa Zlatic (2003): The many faces of agreement, Stanford, CSLI Publications.

Fecha de recepción: 8 de febrero de 2016

Fecha de aceptación: 25 de mayo de 2016 